\title{
SOME REMARKS ON THE MEASURABILITY OF CERTAIN SETS
}

\author{
PAUL ERDÖS
}

The present note contains some elementary remarks on sets defined by simple geometric properties. Our main tool will be the Lebesgue density theorem.

First we introduce a few notations: $d(a, b)$ denotes the distance from $a$ to $b$ and $S(x, r)$ the open sphere of center $x$ and radius $r$. A point $x$ of a set $A$ is said to be of metric density 1 if to every $\epsilon$ there exists a $\delta$ such that $A \cap S(x, r), r<\delta$, has measure greater than $(1-\epsilon)$ times the volume of $S(x, r) . \bar{A}$ denotes the closure of $A$.

(1) Let $E$ be any closed set in $n$-dimensional euclidean space. Denote by $E_{r}$ the set of points whose distance from $E$ is $r(r>0)$. We shall prove that $E_{r}$ has measure 0 .

The set $E_{r}$ is clearly closed and therefore measurable. If it had positive measure it would contain a point of metric density 1 . Let $x$ be any point of $E_{r}$ and $y \in E$ be one of the points in $E$ at distance $r$ from $x$. Then $S(y, r)$ cannot contain any point of $E_{r}$. Thus $x$ cannot be a point of metric density 1 , which completes the proof. This proof is due to T. Radó.

(2) Let $A$ be any set of measure 0 on the positive real axis. Denote by $E_{A}$ the set of points whose distance from $E$ is in $A$. We shall show that $E_{A}$ has measure 0 . As is well known $A$ is contained in a $G_{\delta}$, say $G$ of measure 0 . Thus it suffices to show that $E_{o}$ has measure $0 . E_{g}$ is clearly a $G_{\delta}$ and thus measurable, so that again it will suffice to show that $E_{g}$ has no point of metric density 1 . Let $x$ be any point of $E_{g}$ and $y$ any one of the points of $E$ closest to it. Denote by $C_{x}\left(\eta_{1}, \eta_{2}\right)$ the half cone defined as follows: $z \in C_{x}\left(\eta_{1}, \eta_{2}\right)$ if $d(z, x)<\eta_{1}$ and the angle $z x y$ is less than $\eta_{2}$. Let $R$ be any ray in $C_{x}$ from $x$. Denote by $z$ a variable point of $R$. We assert that if $\eta_{1}$ and $\eta_{2}$ are sufficiently small, $d(z, E)$ is a decreasing function of $d(z, x)$ for which the upper limit of the difference quotient with respect to $d(z, x)$ is less than $-\delta$, with some $\delta>0$. Let $y_{1} \in E$ be one of the points closest to $z$ in $E$. We assert that $d\left(y, y_{1}\right)$ is small if $\eta_{2}$ is small. Clearly by definition $y_{1}$ is contained in $\{S(z, d(z, y))\}$ but not in $S(x, d(x, y))$. Since $d(x, z)<\eta_{1}$ the difference of these two spheres has small diameter if $\eta_{2}$ is small, which shows that $d\left(y, y_{1}\right)$ is small. Now it is geometrically clear that for sufficiently small $\eta_{1}, \eta_{2}$ there exists a $\delta>0$ such that the upper limit of the difference quotient of $d\left(z, y_{1}\right)$ with respect to $d(z, x)$ is less

Received by the editors May 16, 1945, and, in revised form, June 13, 1945. 
than $-\delta$. A fortiori the upper limit of the difference quotient of $d(z, E)$ with respect to $d(z, x)$ is less than $-\delta$. Thus it follows that the set of points on $R$ for which $d(z, E)$ is in $A$ is of measure 0 . Thus by a trivial modification of Fubini's theorem we obtain that $C_{x} \cap E_{g}$ has measure 0 . Thus $x$ could not have been a point of metric density 1 , which completes our proof.

Let $S$ be any measurable set on the positive real axis; it is easy to see that $E_{S}$ is also measurable. For $S$ can be written as $F+A$ where $F$ is an $F_{\sigma}$ and $A$ is of measure 0 . Now clearly $E_{S}=E_{F}+E_{A} . E_{F}$ is measurable since it is also an $F_{\sigma}$ and $E_{A}$ is of measure 0 . Therefore, $E_{S}$ is measurable.

(3) Denote by $M$ the set of those points for which there is more than one closest point in $E$. It is known that the necessary and sufficient condition for $E$ to be convex is that $M$ be empty. We shall prove that $M$ has measure 0 .

For $x \in M$, denote by $\phi(x)$ the set of points closest to $x$. Clearly the set of points $M_{c}$ for which the diameter of $\phi(x)$ is not less than $c$ is closed, thus $M$ is an $F_{\sigma}$ and thus measurable. It suffices to show that $M_{c}$ has measure 0 , or that it can have no point of metric density 1 . Let $y \in \phi(x)$ be arbitrary $\left(\phi(x)\right.$ is of course closed). Define $C_{x}\left(\eta_{1}, \eta_{2}\right)$ as in (2). We shall prove that no point of $C_{x}\left(\eta_{1}, \eta_{2}\right)$ (except $x$ ) belongs to $M_{c}$ and this will show that $x$ cannot have metric density 1 . If $z \in M_{c} \cap C_{x}\left(\eta_{1}, \eta_{2}\right)$ there exists a sphere $S(z, r), r \leqq d(z, y)$, such that $S(z, r)$ contains no points of $E$ in its interior and $\{S(z, r)\}$ contains two points $u$ and $v$ of $E$ with $d(u, v) \geqq c$. But $u$ and $v$ cannot be in the interior of $S(x, d(x, y))$. Hence they must be in [Comp $(S(x, d(x, y))]$ $\cap\{S(z, r)\}$ (Comp $A$ denotes the complement of $A$ ), but for $\eta_{2}=\eta_{2}(c)$ small enough the diameter of this set is less than $c$, which is a contradiction. This completes the proof.

The problems in (1) and (3) were suggested to me by Deane Montgomery.

(4) Let $x$ be any point in the complement of $E$. As before we denote by $\phi(x)$ the set of points in $E$ closest to $x$. We shall prove that $\sum_{x} \bigoplus_{E} \phi(x)$ has measure 0 .

It will be sufficient to prove that no point $z \in \sum_{x} \bigoplus_{E} \phi(x)$ has upper metric density $1 .^{1}$ If $z \in \phi(x)$ then $S(x, d(x, z))$ contains no point of $E$ in its interior (and $\sum_{x} \bigoplus_{E} \phi(x) \subset E$ ), which proves our theorem.

(5) Denote by $M_{k}$ the set of points for which $\phi(x)$ contains $k$ points not all in a $(k-2)$-dimensional euclidean subspace. In (3) we proved that $M_{2}$ has $n$-dimensional measure 0 . I conjecture that $M_{k}$

${ }^{1}$ Let $E$ be any set. Then the upper metric density is 1 at almost all points of $E$. (See, for example, Hildebrandt, Bull. Amer. Math. Soc. vol. 32 (1926) p. 451.) 
has Hausdorff dimension $n+1-k$. At present I can prove this only for $k=n+1$. In fact we shall prove that $M_{n+1}$ is denumerable. For the sake of simplicity we shall restrict ourselves to $n=2$. The proof for the general case is not an easy generalization of the case $n=2$, but we omit details.

Suppose then that $M_{3}$ is nondenumerable. Then it must contain a point of condensation, $x$ say. Put $r=d(x, E)$. There exist nondenumerably many points $z$ such that $r-\epsilon<d(z, E)<r+\epsilon$, and $S(z, d(z, E))$ contains at least three points of $E$ on its boundary. $S(z, d(z, E) \cap \cap E$ is closed. Denote by $t_{z}$ the maximum of the smallest side of all possible triangles formed from points of $S(z, d(z, E)) \cap E$. By a well known argument there exists a constant $c>0$ such that for every $\delta>0$ there are uncountably many points $z$ satisfying

$$
d(z, x)<\epsilon, \quad c \leqq t_{z}<c+\delta .
$$

Choose $\delta$ small, and consider $\bigcup_{z} S(z, d(z, E))$ with $z$ satisfying (1). Denote the boundary of this domain by $B$. Let $p$ be any point of $B$ and denote by $C_{p}(\eta)$ the half cone whose vertex is at $p$ and whose center line is the extension of the line from $x$ to $p$. It is easy to see that for sufficiently small $\epsilon$ there exists an $\eta>0$ such that for any point $p$ on $B, C_{p}(\eta)$ does not contain any point of $B$ other than $p$. From this it can be shown by straightforward methods that $B$ is a rectifiable curve ${ }^{2}$ and hence can contain only countably many arcs of circles. This we shall show to be false. Let $z_{1}$ be any point satisfying (1). Denote by $(a, b)$ the arc on $S\left(z_{1}, d\left(z_{1}, E\right)\right)$ determined by the side of length $t_{z_{1}}$. Since we can choose $z_{1}$ in uncountably many ways, we can assume that $z_{1}$ has been chosen so that the arc $(a, b)$ does not lie on $B$. But since $a \in B$ and $b \in B$ there must exist a point $z_{2}$ satisfying (1) such that $S\left(z_{2},\left(d\left(z_{2}, E\right)\right)\right.$ intersects $S\left(z_{1}, d\left(z_{1}, E\right)\right)$ in two points $u$ and $v$ on the arc $(a, b)$. Therefore if $\delta$ is a sufficiently small fraction of $c$,

$$
t_{z_{2}}<c
$$

which shows that $z_{2}$ does not satisfy (1), an evident contradiction. This completes the proof.

(6) In (1) we proved that $E_{r}$ has $n$-dimensional measure 0 . Let us now assume that $E$ is bounded, then we shall sketch a proof of the fact that $E_{r}$ has finite $(n-1)$-dimensional measure.

Let $D$ be the diameter of $E$. Assume first that $r$ is large. Let $x$ be a fixed point of $E$ and $p$ any point of $E_{r}$. Then it is easy to see that $C_{p}(\eta)$

\footnotetext{
2 Pauc, J. Reine Angew. Math. vol. 185 (1943) pp. 127-128. Pauc proves a more general theorem.
} 
does not contain any point of $E_{r}$ other than $p . C_{p}(\eta)$ is defined as in (5). From this it can be shown that $E_{r}$ has finite $(n-1)$-dimensional measure. Let us not assume now that $r$ is large. We then write $E=\bigcup_{k=1}^{m} E^{(k)}$ where the $E^{k}$ s are closed and their diameter is less than $\epsilon$. Then, by what has been shown before, if $\epsilon$ is small enough $E_{r}^{(\boldsymbol{k})}$ has finite $(n-1)$-dimensional measure. Clearly $E_{r} \subset \bigcup_{k=1}^{m} E_{r}^{(k)}$. But $E_{r}$ is closed, therefore its $(n-1)$-dimensional measure exists, and it clearly can not be 0 , since it separates the space. Thus $E_{r}$ must have finite $(n-1)$-dimensional measure.

Added in proof. The author has recently discovered that the following two theorems have been stated by C. Pauc, Revue Scientifique vol. 77 (1939) no. 8: Let the set $E$ be in the plane then $M_{2}$ is contained in the sum of countably many Jordan curves and $M_{3}$ is countable.

UNIVERSITY OF MICHIGAN

\section{REMARK ON TAYLOR'S FORMULA}

Taylor's formula

$$
\text { PHILIP HARTMAN }
$$

$$
f(a)=\sum_{k=0}^{n-1} f^{(k)}(0) a^{k} / k !+f^{(n)}(\xi) a^{n} / n !, \quad 0<\xi<a,
$$

is usually proved under the assumptions that

(I) $f(x)$ is continuous on the closed interval $[0, a]$;

(II) $f(x)$ possesses $n-1$ derivatives on the half closed interval $[0, a)$;

(III) $f^{(n-1)}(x)$ is continuous at $x=0$; and

(IV) $f(x)$ has an $n$th derivative on the open interval $(0, a)$.

In the case $n=1$, the assumption (III) that $f^{(0)}(x) \equiv f(x)$ be continuous at $x=0$ is essential but is contained in condition (I). In the case $n>1$, it will be shown below that the assumption (III) is entirely superfluous, so that (1) is valid whenever (I), (II) and (IV) hold.

The proof of (1) is usually reduced to an application of the mean value theorem to the $(n-1)$ th derivative of $f(x)$ on an interval $[0, c]$, $0<c<a$. Thus, to prove the italicized statement, it is sufficient to show that if $f(x)$, defined on the interval $[0, a]$, is the derivative of a function and $f(x)$ itself possesses a derivative on the open interval $(0, a)$, then there exists a number $\xi$ such that

Received by the editors May 18, 1945. 